\title{
LA CONSTRUCCIÓN SOSTENIBLE. EL ESTADO DE LA CUESTIÓN
}

\author{
(THE CONSTRUCTION SUSTAINABLE. THE STATE OF THE ART)
}

Pere Alavedra, Dr. Ing. Industrial (UPC); Javier Domínguez, Dr. Ing. Industrial (UZ); Engràcia

Gonzalo, Ing. Industrial (UPC) y Javier Serra, Arquitecto (Ministerio de Fomento)

ESPAÑA

fecha de recepción: $27-\mathrm{VI}-97$

\section{RESUMEN}

En este trabajo se expone el estado actual de la cuestión en referencia a la integración de los vocablos de Construcción y Desarrollo Sostenible. Por ello, se expone una reflexión sobre construcción de los edificios sostenibles, en lo que se refiere a la conservación de la energía y de los recursos naturales, la reutilización de estos recursos, la gestión del ciclo de vida, tanto de los edificios como de los materiales y componentes utilizados y las consideraciones a destacar en cuanto a la calidad de las edificaciones, en lo que se refiere a materiales, edificaciones y ambiente urbanizado. Las consideraciones especificadas se refieren tanto a aspectos concernientes a los materiales como a tecnologias utilizados para obtener una mayor eficiencia energética de los edificios y las técnicas de construcción.

\section{S'UMMARY}

This work explains the state of the art regarding the integration of new vocabulary for Construction and Sustainable Development. For this purpose it states an argumentation about construction of sustainable buildings regarding energy-saving and natural resources preservation reutilization of resources, management of buildings life-cycle including all the components, and some considerations are pointed out as for quality not only of materials and buildings, but also of urban environment. These considerations refer both to materials and to the technology employed for a good energetic efficiency of the buildings and of the construction techniques.

\section{Introducción}

Resulta evidente que con el actual ritmo de crecimiento demográfico, a pesar de la disminución en los últimos años de la tasa de crecimiento, continuamos creciendo, año tras año, a una velocidad que podría llegar a duplicar la población humana mundial antes de mediados del siguiente siglo. La actual utilización de los recursos naturales y del medio ambiente supone una disminución del potencial de estos recursos para las generaciones futuras (Xercavins i Valls, 1996).

Fenómenos, como pueden ser el cambio climático y la acentuación del deterioro de la capa de ozono, la aparición de la lluvia ácida, la desforestación o la pérdida de biodiversidad, están causadas por las actividades económicas que tienen lugar actualmente.
Es un error habitual atribuir exclusivamente a la industria y a los sistemas de transporte, especialmente el automóvil, el origen principal de la contaminación.

El entorno construido, donde pasamos más del $90 \%$ de la nuestra vida, es, en gran medida, culpable de dicha contaminación.

Los edificios consumen entre el 20 y el $50 \%$ de los recursos físicos, según su entorno, teniendo especial responsabilidad en el actual deterioro del medio ambiente, la ampliación del parque construido.

Dentro de las actividades industriales, la actividad constructora es la mayor consumidora, junto con la industria asociada, de recursos naturales como pueden ser madera, minerales, agua y energía. Asimismo, los edificios, una vez 
construidos, continúan siendo una causa directa de contaminación ,por las emisiones que se producen en los mismos o el impacto sobre el territorio, creando un ambiente físico alienante y una fuente indirecta, por el consumo de energía y agua necesarios para su funcionamiento.

La construcción de los edificios comporta unos impactos ambientales que incluyen la utilización de materiales que provienen de recursos naturales, la utilización de grandes cantidades de energía, tanto en lo que atiende a su construcción, como a lo largo de su vida y el impacto ocasionado en el emplazamiento. El material, fuertemente manipulado y que ha sufrido un proceso de fabricación, utilizado en el campo de la construcción, tiene unos efectos medioambientales muy importantes, con un contenido muy intensivo en energía.

No se pueden olvidar los costes ecológicos que suponen tanto la extracción de los recursos minerales (canteras, minas, etc.) como la deposición de los residuos originados, que abarcan desde las emisiones tóxicas al envenenamiento de las aguas subterráneas por parte de los vertedores. La construcción y el derribo de los edificios originan una gran cantidad de residuos.

El reciclaje y la reutilización de los residuos de demolición y de los residuos originados en la construcción es una solución que acabará parcialmente con el importante impacto ambiental que tiene su origen en el vertido y la incineración.

Muchos edificios modernos crean atmósferas interiores insalubres y/o peligrosas para sus ocupantes $y$, en una parte significativa de los edificios nuevos o rehabilitados, aparece el denominado "síndrome del edificio enfermo". Los nuevos edificios herméticos con climatización controlada retienen compuestos orgánicos volátiles ( $\mathrm{COV}$ ) que pueden llegar a unas concentraciones centenares de veces más altas que en el exterior.

La aplicación de los criterios de sostenibilidad y de una utilización racional de los recursos naturales disponibles en la construcción requerirá realizar unos cambios importantes en los valores, que ésta tiene como cultura propia. Estos criterios o, más correctamente, principios de sostenibilidad llevarán hacia una conservación de los recursos naturales, una maximización en la reutilización de los recursos, una gestión del ciclo de vida, así como reducciones de la energía utilizada.

Múltiples son las actuaciones políticas que sobre este tema se han llevado a cabo, tanto a nivel nacional como internacional. Así, en los 15 puntos de que consta la Redacción de Berlín, salida de la conferencia de Berlín sobre el desarrollo urbano sostenible celebrada, en aquella ciudad, del 19 al 21 de marzo de 1996, se hace especial hincapié en los temas que abarca el presente trabajo.
La argumentación española en la $1^{\mathrm{a}}$ Conferencia Europea de Ministros sobre Política de Vivienda Sostenible, celebrada en Copenhague los días 22 y 23 de abril de 1996, se fundamentó en: "la necesidad de recuperar el concepto de ciudad próspera y cohesionada de forma que, mejorando su integración en el territorio y el medio natural, se reduzca su impacto ambiental.

Por tanto, debe aproximarse la regeneración urbana y, por ello, favorecer la reutilización del parque de viviendas existente y con ellos mejorar su eficiencia energética y medioambiental, considerando la vivienda, no como un elemento aislado, sino intrínsecamente inseparable de su entorno e interrelacionada con la política de suelo en el marco de la construcción de la ciudad".

El comunicado final de dicha conferencia ministerial hacia un especial hincapié en los siguientes puntos:

1. Planeamiento Urbano

2. Reducción de las demandas derivadas del transporte

3. Ahorro de agua

4. Ahorro energético

5. Tratamiento de los desechos de forma especial los materiales de construcción

6. Mejora del clima interior de los edificios

7. Desarrollo de la implicación local en los procesos de planeamiento y gestión de la vivienda

8. Mantenimiento y más rehabilitación de las viviendas existentes

9. Utilización de nuevos materiales constructivos bajo el concepto de Sostenibilidad

10. Libertad de circulación de los materiales

La Conferencia atendió la propuesta de la Comisión Europea de que los principales objetivos eran:

- Cambiode información sobre lasinvestigaciones relevantes

- Análisis sobre el Ciclo de Vida

- La normalización sobre los desempeños energéticos

- La cooperación internacional sobre los sistemas estadísticos y en indicadores claves

- Información sobre la iniciativa nacional

\section{Definición de Sostenibilidad}

La Sostenibilidad consiste en la adaptación del entorno de los seres humanos a un factor limitante: la capacidad del entorno de asumir la presión humana de manera que sus recursos naturales no se degraden irreversiblemente (Cáceres, 1996).

Es necesario recordar los tres principios básicos que, formulados por el economista Herman Daly, nos permiten avanzar, medioambientalmente hablando, hacia un desarrollo sostenible: 
1. Para una fuente de recursos renovable, no consumirla a una velocidad superior a la de su renovación natural

2. Para una fuente no renovable, no consumirla sin dedicar la parte necesaria de la energía resultante en desarrollar una nueva "fuente" que, agotada la primera, nos permita continuar disfrutando de las mismas prestaciones.

3. Para un residuo, no generar más que aquél que el sumidero correspondiente sea capaz de absorber e inertizar de forma natural (Xercavins i Valls, 1996).

\section{La Construcción Sostenible}

\subsection{Definiciones de Construcción Sostenible}

Partiendo de diversos autores, se recogen a continuación algunas definiciones del término "Construcción Sostenible”, que, asumidas globalmente, nos aportan una buena comprensión de la idea que comportan.

La Construcción sostenible, que debería ser la construcción del futuro, se puede definir como aquélla que, con especial respeto y compromiso con el Medio Ambiente, implica el uso sostenible de la energía. Cabe destacar la importancia del estudio de la aplicación de las energías renovables en la construcción de los edificios, así como una especial atención al impacto ambiental que ocasiona la aplicación de determinados materiales de construcción y la minimización del consumo de energía que implica la utilización de los edificios (Casado, 1996).

La Construcción Sostenible se dirige hacia una reducción de los impactos ambientales causados por los procesos de construcción, uso y derribo de los edificios y por el ambiente urbanizado (Lanting, 1996).

El término de Construcción Sostenible abarca, no sólo a los edificios propiamente dichos, sino que también debe tener en cuenta su entorno y la manera como se comportan para formar las ciudades. El desarrollo urbano sostenible deberá tener la intención de crear un entorno urbano que no atente contra el medio ambiente, con recursos, no sólo en cuanto a las formas y la eficiencia energética, sino también en su función, como un lugar para vivir (WWF, 1993).

La Construcción Sostenible deberá entenderse como el desarrollo de la Construcción tradicional, pero con una responsabilidad considerable con el Medio Ambiente por todas las partes y participantes. Ello implica un interés creciente en todas las etapas de la construcción, considerando las diferentes alternativas en el proceso de construcción, en favor de la minimización del agotamiento de los recursos, previniendo la degradación ambiental o los perjuicios y proporcionando un ambiente saludable, tanto en el interior de los edificios como en su entorno (Kibert, 1994).

\subsection{Aspectos a considerar en la Construcción Sostenible}

La sostenibilidad tendrá en cuenta no sólo la construcción en la creación del ambiente, sino también los efectos que ésta producirá en aquéllos que lo llevan a cabo y en los que vivirán en ellos. La importancia creciente en las consideraciones del "síndrome del edifício enfermo" en los edificios de oficinas y la "sensibilidad ambiental" en la construcción de viviendas ha dado lugar a una mayor consideración de los efectos que los materiales de construcción tienen en la salud humana (Vale et al. 1993).

Se tratará de construir en base a unos principios, que podríamos considerar ecológicos y que se enumeran a continuación (Kibert, 1994):

- Conservación de los recursos

- Reutilización de recursos

- Utilización de recursos reciclables y renovables en la construcción

- Consideraciones respecto a la gestión del ciclo de vida de las materias primas utilizadas, con la correspondiente prevención de residuos y de emisiones

- Reducción en la utilización de la energía

- Incremento de la calidad, en lo referente a materiales, edificaciones y ambiente urbanizado

- Protección del Medio Ambiente

- Creación de un ambiente saludable y no tóxico en los edificios (Lanting, 1996)

Los recursos disponibles para llevar a cabo los objetivos de la Construcción Sostenible son los siguientes:

- Energía, que implicará una efíciencia energética y un control en el crecimiento de la movilidad

- Terreno y biodiversidad. La correcta utilización del terreno requerirá la integración de una política ambiental y una planificación estricta del terreno utilizado. La construcción ocasiona un impacto directo en labiodiversidad a través de la fragmentación de las áreas naturales y de los ecosistemas

- Recursos minerales, que implicará un uso más eficiente de las materias primas y del agua, combinado con un reciclaje a ciclo cerrado

La definición de Construcción Sostenible lleva asociada tres verbos: reducir, conservar y mantener. La combinación de los principios ecológicos y de los recursos disponibles nos proporcionan una serie de consideraciones a tener en cuenta.

La reducción en la utilización de los recursos disponibles se llevará a cabo a través de la reutilización, el reciclaje, la utilización de recursos renovables y un uso eficiênte de los recursos. Se tratará de incrementar la vida de los productos utilizados, un incremento en la eficiencia energética y 
del agua, así como un uso multifuncional del terreno (Lanting, 1996).

La conservación de las áreas naturales y de la biodiversidad se llevará a cabo a partir de la restricción en la utilización del terreno, una reducción de la fragmentación y la prevención de las emisiones tóxicas.

El mantenimiento de un ambiente interior saludable y de la calidad de los ambientes urbanizados se llevará a cabo a través de la utilización de materiales con bajas emisiones tóxicas, una ventilación efectiva, una compatibilidad con las necesidades de los ocupantes, previsiones de transporte, seguridad y disminución de ruidos, contaminación y olores (Lanting, 1996).

A partir de la información anterior, se podrían enumerar a grandes rasgos los requisitos que deberían cumplir los edificios sostenibles:

- Consumir una mínima cantidad de energía y agua a lo largo de su vida

- Hacer un uso eficiente de las materias primas (materiales que no perjudican el medio ambiente, materiales renovables y caracterizados por su desmontabilidad)

- Generar unas mínimas cantidades de residuos y contaminación a lo largo de su vida (durabilidad y reciclabilidad)

- Utilizar un mínimo de terreno, integrándose correctamente en el ambiente natural

-Adaptarse a las necesidades actuales y futuras de los usuarios (flexibilidad, adaptabilidad y calidad del emplazamiento)

- Crear un ambiente interior saludable (Lanting, 1996)

\section{Los edificios y la Sostenibilidad}

El objetivo principal de los edificios ha sido la de proteger a sus ocupantes de los elementos naturales. Los principales esfuerzos se han enfocado a la mejora de los aspectos necesarios para llevar a cabo este objetivo, es decir, una mejora en la calidad global del edificio y en el control de los costes correspondientes.

Actualmente la noción de Desarrollo Sostenible introduce una restricción adicional, que es la de cumplir el objetivo principal de los edificios sin comprometer la posibilidad de las generaciones futuras de satisfacer sus necesidades (Bourdeau, 1996).

Los edificios, alo largo de su construcción, usoy demolición ocasionan una gran cantidad de impactos ambientales que nacen de nuestra actividad económica. Éstos ocasionan un gran impacto en el ambiente global a través de la energía utilizada para proveer a los edificios de los servicios necesarios y de la energía contenida en los materiales utilizados en la construcción. Los edificios son responsables de aproximadamente el $50 \%$ de energía utilizada y de las emisiones de $\mathrm{CO}_{2}$ a la atmósfera. El ambiente interior tiene un mayor impacto en la salud y el confort.Otros aspectos incluyen el adelgazamiento de la capa de ozono como resultado de la masiva utilización de productos químicos, como pueden ser los clorofluorocarbonados (CFC's), hidroclorofluorocarbonados(HCFC's) y halones, utilizados comúnmente como refrigerantes, etc. (Baldwin, 1996).

\subsection{El Impacto Ambiental de los edificios}

Deberán tenerse en cuenta los impactos ambientales de los edificios y de sus materiales, antes, durante y después de su construcción. Los diferentes efectos se calcularán con el coste de adoptar nuevas alternativas prácticas (WWF, 1993).

Los flujos de materia o energía que entran o salen del sistema estudiado contribuyen, de forma diferenciada, a un cierto número de impactos, o defectos (globales), sobre el medio ambiente. Se puede citar el efecto invernadero (o contribución al recalentamiento global), la acidificación atmosférica (o la lluvia ácida), la destrucción de ozono estratosférico, la eutrofización, el agotamiento de los recursos naturales (Moch, 1996).

\subsubsection{Los efectos de los materiales sobre el Medio Ambien-} te

Evaluar la dimensión medioambiental de un producto de construcción es intentar calificar y cuantificar el pesode los impactos que se le asocian por el conjunto de su ciclo de vida, desde la extracción de las materias primas hasta el final de su vida (Moch, 1996).

El proceso de fabricación de los materiales de construcción, así como de los productos de los cuales muchos están formados, ocasiona un impacto ambiental. Este impacto tiene su origen en la extracción de los recursos naturales necesarios para su elaboración, incluyendo el proceso de fabricación y el consumo de energía, que se deriva en emisiones tóxicas a la atmósfera, que resultan contaminantes, corrosivas y altamente perjudiciales para la salud. Lo que se pretende con la aplicación de los criterios de la construcción sostenible es la construcción de edificios con una disminución de estos materiales y evitar, siempre y cuando sea posible, la utilización de sustancias que, al final de su ciclo de vida, originen residuos peligrosos.

Los principales efectos que los materiales utilizados en la construcción provocan sobre el Medio Ambiente son los siguientes:

- Consumo energético

- Producción de residuos sólidos

- Incidencia en el efecto invernadero 
- Incidencia en la capa de ozono

- Otros factores de contaminación ambiental (Casado, 1996).

4.1.2. Estrategia de reducción al mínimo del impacto ambiental de los materiales de construcción.

Una estrategia óptima para minimizar el impacto ambiental sería aquélla que utilizase soluciones que redujesen al mínimo, de manera equilibrada, los efectos que éstos producen sobre el Medio Ambiente, es decir, sobre el consumo de energía, la producción de residuos y la contaminación (Speare, 1995)

- Utilización de materiales reciclables para la producción de los áridos del hormigón, en lugar de utilizar materias primas naturales

- Reciclaje de materiales: reutilización de la madera, utilización de materiales reciclados/reutilizados en la construcción de las paredes, techos y suelos; uso de residuos industriales en algunos materiales (Baldwin, 1996)

Cabe destacar que la madera es un recurso natural renovable, que consume poca cantidad de energía en su proceso de transformación como material de construcción, pero los tratamientos de conservación y protección que se apliquen pueden originar emisiones y residuos tóxicos.

- Reutilización de los residuos de otras construcciones o demoliciones, en un nivel de alta calidad y que no sean utilizados en aplicaciones de baja importancia o vertidos en los vertederos (Speare, 1995)

El impacto ambiental debido al transporte de los materiales supone un coste indirectoen términos de contaminación, en cuanto a las emisiones de $\mathrm{CO}_{2}$ producidas por los gases de escape.

Las pinturas, disolventes y los tratamientos realizados a la madera plantean importantes riesgos para la salud humana y los perjuicios que supone al ambiente a lo largo de su producción, uso y disposición final (WWF, 1993).

El diseño del edificio y la elección de los materiales se realizará teniendo en cuenta una reducción al mínimo en la cantidad de materiales que liberen sustancias químicas peligrosas y la incorporación de materiales y componentes con un bajo índice de ODP (ozone depletion potential) (Baldwin, 1996)

4.1.3. Reducción al mínimo de los consumos energéticos en la utilización de las construcciones.

Desde la proyección de los edificios se puede controlar en gran medida su consumo energético. Posteriormente, en la utilización de los edificios tendrá una gran importancia la gestión de la energía, la intervención de los usuarios y el mantenimiento.

La implantación de los edificios juega un papel fundamental en el consumo de energía. No siempre se pueden escoger las condiciones más favorables, pero la referencia al clima, la vegetación, la topografía y el tejido edificado tienen que ser un primer paso, tanto si lo aprovechamos como si nos tenemos que proteger de las condiciones adversas (Casado, 1996).

Para llevar a cabo un uso eficiente de la energía y de su conservación se tendrán que considerar los siguientes aspectos en la construcción de los edificios:

- Aislamiento y ventilación

- Sistemas de control de la energía en los edificios y otros controles automáticos

- Uso de monitores y gestores energéticos

- Control por ordenador de la iluminación, temperatura y condiciones climáticas

- Desarrollo en aplicaciones de baja energía y tecnologías limpias

- Fuentes de energía renovable

- Diseño basado en un consumo bajo de energía y planificación para una eficiencia energética (WWF, 1993).

\subsubsection{Impacto en la Planificación de la Localización.}

Es en el planeamiento urbanístico el ámbito en el cual se pueden conseguir las mejores aportaciones del ambiente en la edificación, ya que puede conducir a un ambiente más saludable y agradable (Casado, 1996)

Un estudio de Planeamiento tendrá en cuenta los siguientes aspectos:

- Existencia de paisaje, importancia ecológica y arquitectónica de la localización

- Valoración del impacto ambiental

- Determinación previa de los posibles usos del transporte

- Previsión de zonas seguras para el almacenamiento de productos y residuos en el lugar de construcción y para la disposición final de residuos (WWF, 1993)

- Impactos en el proceso de construcción, como pueden ser un incremento en la cantidad de transporte, polvo y ruidos

\subsection{La calidad en la edificación}

La calidad en la edificación es la clave para relanzar el mercado, mejorar las condiciones medioambientales y ahorrar recursos energéticos. Esta visión incluye tanto los materiales, como los sistemas o estrategias urbanas que 
inciden sobre la calidad. Entre las propuestas que contribuirán a mejorar la calidad podemos mencionar las siguientes:

- Limitar el riesgo económico que supone actualmente la compra de inmuebles y promocionar las inversiones en proyectos de construcción de alta calidad

- Crear el etiquetado ecológico, tanto para edificios como para productos de construcción y favorecer un mecanismo de mercado que promueva el cambio hacia esta realidad

- Reducir los costes constructivos e introducir el concepto del menor coste posible en el mantenimiento del inmueble

- Incrementar la estandarización de los diferentes componentes de la construcción y mejorar la diseminación de aquellas tecnologías y sistemas de interés general

- Desarrollar sistemas apropiados de control de calidad, adaptados a las necesidades de los constructores y diseñadores y orientados a promocionar una garantía en los resultados energéticos del edificio (Landabaso, 1996)

\subsection{La calidad del ambiente interior}

Los materiales y los componentes con los que se construye el edificio pueden ser una fuente de materiales problemáticos, como pueden ser los tratamientos químicos aplicados a diferentes materiales, que se evaporan en la atmósfera del edificio. Los sistemas de control ambiental y otros servicios incrementan la comodidad y salubridad de los ambientes en los edificios (Groak, 1992).

Actualmente se está investigando en la reducción y eliminación de las emisiones de los productos químicos contenidos en los diferentes materiales y otras aplicaciones para mejorar la calidad del ambiente interior (Canada Mortage, 1993.).

Se pueden considerar los siguientes aspectos en cuanto a la calidad del ambiente interior:

- Caracterizar las fuentes de contaminación y los elementos contaminantes del aire

- Optimización de los equipos de ventilación

- Clarificar los aspectos sociológicos relacionados con la calidad del aire

- Controlar los elementos contaminantes del aire.

- Desarrollar una estandarización en este aspecto (Groak, 1992)

\section{Conclusiones}

La experiencia de los últimos veinte años ha demostrado que no resulta fácil cambiar el sistema de construcción de los edificios y su funcionamiento. Para lograr una Construcción Sostenible debe romperse con la rutina y los malos hábitos adquiridos por décadas de derroche de los recursos naturales.
Deberá cambiarse la mentalidad de la industria y de las estrategias económicas con la finalidad de que den prioridad al reciclaje ante la tendencia tradicional de la extracción de materias naturales. Deberá fomentarse la utilización de sistemas constructivos y energéticos en base a productos y energías renovables.

Es en este entorno en el que la humanidad toma conciencia de la importancia, cada día más evidente, de que los aspectos medioambientales tendrán consecuencias muy importantes en las principales opciones del proceso constructivo.

\section{BIBLIOGRAFÍA}

(1) BALDWIN, ROGER (1996): Environmental Assessment and Management of Buildings. The UK viewpoint, Report 7150/1 for consultation, BSRIA.

(2) BOURDEAU, LUC (1996): Environment and Buildings in France, CIB W82 Comission Meeting. Centre Scientifique et Technique du Bâtiment (CSTB).

(3) CÁCERESTERAN, JOHANNA(1996): Desenvolupament Sostenible. Revista Tracte, Número 66, Octubre del 1996. ISSN 1132-7081.

(4) CASADO MARTÍNEZ, N. (1996): Edificios de Alta Calidad Ambiental, Ibérica, Alta Tecnología, ISSN 0211-0776.

(5) CANADA MORTAGE ANDHOUSING CORPORATION (1993) :The Clean Air Guide: How to Identify and Correct Indoor air Problems in your home, Ottawa.

(6) Communique of 1 st European Minister Conference on Sustainable Housing Policies (April 22-23, 1996).

(7) KIBERT, CHARLES et al.(1994): CIB-TG16, First International Conference on Sustainable Construction, Florida.

(8) KILFORD, STEVEN: (1996). Sustainable Construction, The UK viewpoint, The BRSIA Report. CIBW82.

(9) LANDABASO, ÁNGEL (1996): Eficiència Energètica a l'edificació; Estat actual de les diferents tecnologies, I Jornades: Construcció i Desenvolupament Sostenible, Barcelona,16, 17 i 18 de maig de 1996.

(10) LANTING, ROEL (1996): Sustainable Construction in The Netherlands -A perspective to the year 2010. Working paper for CIB W82 Future Studies in Construction. TNO Bouw Publication number 96-BKR-P007.

(11) MOCH, YVES (1996): Impacte Ambiental dels materials de construcció, I Jornades Construcció i Desenvolupament Sostenible, Barcelona, 16, 17 i 18 de maig de 1996 
(12) SPEARE, R.S. (1995): Recycling of structural Materials, The Structural Engineer, Volume 73, Nº 13, 4 July 1995.

(13) GROAK, STEVEN (1992): The analisis of sensations, The analysis of building, E\&FN SPON.

(14) VALE, B. I VALE, R. (1993): The Untapped Potential of the Low-Energy Building, TOWN \& PLANNING, Vol. 62 .

(15) WWF:(1993): The Built Environment Sector, Pre-Seminair
(16) Report. Council for Environmental Education WWF Department of Environment, De Monfort University Leicester.

(17) XERCAVINS I VALLS, JOSEP (1996): Què és el desenvolupament sostenible?, I Jornades: Construcció i Desenvolupament sostenible, Barcelona, 16,17, i 18 de maig de 1996.

(18) The Berlin Declaration by the Berlin Conference on Sustainable Urban Development (March 19-21, 1996).

\section{Publicaciones del Instituto Eduardo Torroja-CSIC}

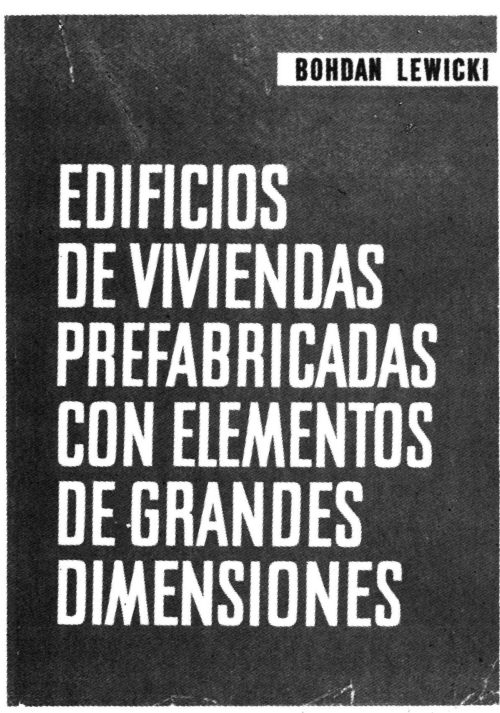

Bohdan Lewicki

Este libro trata de los problemas relativos a la construcción de los edificios de viviendas o publicos realizados con elementos prefabricados de grandes dimensiones. Se han estudiado los problemas de arriostramiento asi como los que plantea la resistencia de examinado las cuestiones de orden higrotermico, acústico y de resistencia al fuego; también se ha profundizado en el estudio de la estanquidad de los muros exteriores y de las juntas.

La obra incluye numerosas ilustraciones que dan detalles de diversas soluciones, as como ejemplos de cálculo, tablas de valores numéricos, diagramas y ábacos.

Un volumen encuadernado en tela, de $24 \times 17 \mathrm{~cm}$, compuesto de 616 págs.

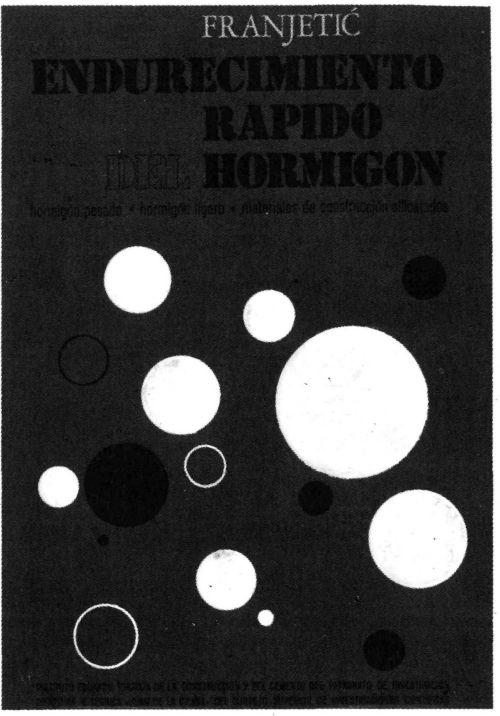

Zorislav Franjetić

En la obra de Franjetić se expone de una forma minuciosa, ordenada y sistemática, todo un cuerpo de doctrina que reúne el conocimiento actual sobre el endurecimiento rápido del hormigón. Parte el autor de los principios básicos y llega a las últimas conprincipios básicos y llega a las últimas con-
secuencias y realidades técnicas y economicas.

Es una obra de consulta, tanto para el investigador sobre la materia, como para el proyectista y el realizador y montador de proyectista y el realizador y montador de
plantas e instalaciones y equipos de curado y endurecimiento rápido.

Un volumen encuadernado en cartóné, de $17 \times 24,5 \mathrm{~cm}$, compuesto de 385 págs. 110 figuras y 10 tablas.

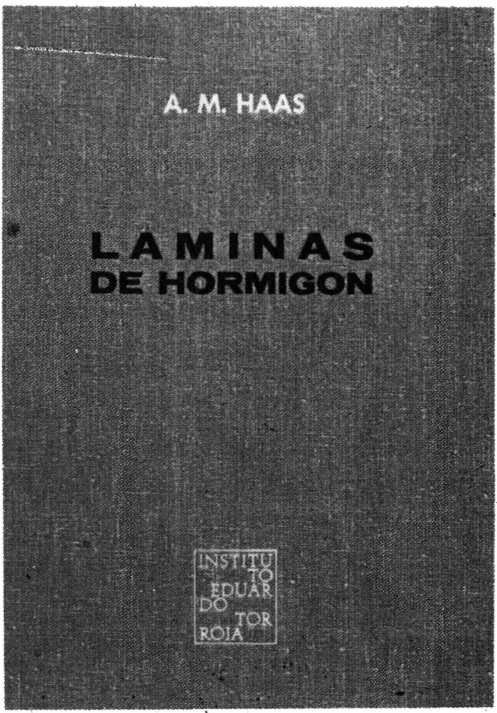

\section{A. M. Haas}

Al escribir este libro el autor intento poner a disposición de los estudiantes y de los ingenieros unos conocimientos prácticos, adecuados para servir de guia en el diseño y construcción de láminas delgadas de hormigón.

El autor está convencido de que el éxito en el diseño de una lámina exige, por parte del proyectista, un examen de las tres fases por las que pasa la materialización de la lámina: el diseño, el análisis estructural y la construcción de la estructura.

Un volumen encuadernado en-tela, de $17 \times 24,5 \mathrm{~cm}$, compuesto de 420 págs., 141 figuras, 22 fotografías y 6 tablas. 